\title{
Avaliação de genótipos de bananeira em Goiânia, estado de Goiás ${ }^{1}$
}

\author{
Evaluation of banana genotypes in Goiânia, state of Goiás
}

\author{
Khaico Henrique Mendonça ${ }^{2}$, Diogo Alisson dos Santos Duarte ${ }^{2}$, Vinicyus Americo de Melo Costa ${ }^{2}$, Glays \\ Rodrigues Matos ${ }^{3}$ e Alexsander Seleguini ${ }^{4 *}$
}

\begin{abstract}
RESUMO - No Brasil, 60\% da área cultivada com bananeiras são das cultivares Prata, Prata Anã e Pacovan, no entanto, apesar da representatividade e importância destes genótipos, todos são suscetíveis às principais pragas e doenças desta Cultura. O objetivo foi avaliar o crescimento, desenvolvimento e produção de 23 genótipos de bananeiras: Caipira, Calipso, Bucaneiro, FHIA-02, FHIA-17, Thap Maeo, FHIA-01, FHIA-18, PA42-44, PA94-01, ST42-08, PV42-53, PV42-142, e PV79-34, PV94-01, PV42-81, YB42-21, YB42-03, YB42-07 Pacovan, Prata Anã, Maçã e Grand Naine, no município de Goiânia-GO, visando incorporar genótipos aos sistemas de produção comercial na região. O experimento foi conduzido na Embrapa Transferência de Tecnologia - Escritório de Negócios de Goiânia. Adotou-se o delineamento experimental em blocos ao acaso, com três repetições. As características avaliadas foram: altura da planta, circunferência do pseudocaule, número de folhas vivas na floração e na colheita, ciclo para florescimento e colheita do primeiro cacho, número de pencas por cacho, massa dos frutos, massa do cacho e das pencas, número, comprimento e diâmetro dos frutos. Os dados foram submetidos à análise de variância e as médias comparadas pelo teste de Scott-Knott a 5\%. Os resultados obtidos neste trabalho qualificam as cultivares FHIA 17, Tropical, FHIA 01, Grand Naine e Bucaneiro como promissoras para serem incorporadas aos sistemas de produção da região.
\end{abstract}

Palavras-chave: Musa spp.. Cultivares. Seleção. Produção.

\begin{abstract}
In Brazil, 60\% of the area planted with banana are of the Prata, Prata Anã and Pacovan cultivars, However, despite the importance and representativeness of these genotypes they are all susceptible to the major pests and diseases of this crop. The objective here was to evaluate the growth, development and yield of 23 banana genotypes: Caipira, Calipso, Bucaneiro, FHIA-02, FHIA-17, Thap Maeo, FHIA-01, FHIA-18, PA42-44, PA94-01, ST42 -08, PV4253, PV42-142-34 and PV79, PV94-01, PV42-81, YB42-21-03 YB42, YB42-07 Pacovan, Prat, Prata Anã, Maçã and Grand Naine in the city of Goiânia, in the state of Goiás, with a view to matching genotypes to commercial production systems in the region. The experiment was carried out at Embrapa Technology Transfer at the Goiânia Business Office. An experimental design of randomized blocks with three replications was adopted. The following characteristics were evaluated: plant height, pseudostem circumference, number of living leaves at flowering and at harvest, flowering and harvest cycle of the first bunch, number of hands per bunch, fruit weight, bunch and hand weight, fruit number, length and diameter. Data underwent variance analysis and the means were compared by the Scott-Knott test at 5\%. The results of this work qualify the cultivars FHIA 17, Tropical, FHIA 01, Grand Naine and Buccaneer as promising for incorporation into the production systems of the region.
\end{abstract}

Key words:Musa spp.. Cultivars. Selection. Production.

\footnotetext{
* Autor para correspondência

${ }^{1}$ Recebido para publicação em 20/09/2011; aprovado em 28/12/2012

Parte do projeto financiado pela Embrapa - Chamada 002/2007

${ }^{2}$ Escola de Agronomia e Engenharia de Alimentos, Universidade Federal de Goiás, Goiânia-GO, Brasil, khaicoufg@gmail.com, diogoalisson@ gmail.com, vinicyus.americo@ hotmail.com

${ }^{3}$ Embrapa Transferência de Tecnologia, Escritório de Negócios de Goiânia, Goiânia-GO, Brasil, glays.matos@hotmail.com

${ }^{4}$ Setor de Horticultura, Escola de Agronomia e Engenharia de Alimentos, Universidade Federal de Goiás, Goiânia-GO, Brasil, aseleguini@gmail.com
} 


\section{INTRODUÇÃO}

No Brasil, a cultura da banana ocupa o segundo lugar em volume de frutas produzidas. Entre as frutas mais consumidas nos domicílios das principais regiões metropolitanas do país, a banana só é superada pela laranja. Presente nas mais diversas camadas da população, a banana aparece na mesa dos brasileiros não apenas como sobremesa, mas como alimento, com um consumo per capita em torno de $25 \mathrm{~kg} \mathrm{ano}^{-1}$ (GASPAROTTO; PEREIRA, 2010).

O Brasil é o quarto maior produtor mundial de bananas, com 7,11 milhões de toneladas em uma área cultivada de 513.656 ha, sendo os principais estados produtores São Paulo, Bahia, Santa Catarina, Minas Gerais e Pará. No estado de Goiás, a bananeira apesar de ser a principal frutífera em produção (173,6 mil toneladas) e área plantada (12.6 mil ha) (INSTITUTO BRASILEIRO DE GEOGRAFIA E ESTATÍSTICA, 2011) faltam pesquisas e incentivos para o fortalecimento da cadeia de produção da fruta no Estado. As cultivares mais exploradas no Brasil são a Prata, a Pacovan, a Prata Anã, a Maçã, a Mysore, a Terra e a D'Angola, do grupo genômico AAB, utilizadas unicamente para o mercado interno, e a Nanica, Nanicão e Grande Naine, do grupo AAA, usadas, principalmente, no mercado para exportação. As cultivares Prata, Prata Anã e Pacovan são responsáveis por quase $60 \%$ da área cultivada no Brasil. No entanto, apesar da representatividade e importância destes genótipos, todos são suscetíveis às principais pragas e doenças da bananeira, que podem ocasionar perdas na produção de até $100 \%$, de acordo com as práticas culturais realizadas e as condições ambientais (SILVA; BOLIANI; CORRÊA, 2006).

Embora o Estado de Goiás não possua indícios da presença da Sigatoka-negra (Mycosphaerella fijiensis Morelet.), hoje considerada a principal doença dos bananais nacionais, os produtores devem estar preparados e tomar as medidas sanitárias necessárias para evitar a introdução da doença, bem como, a utilização de material varietal resistente, imprescindíveis para o sucesso do sistema de produção da cultura. Segundo Cordeiro et al. (2005) a Sigatoka-negra é uma doença de difícil controle, e sua prevenção vem sendo feita com a obtenção de mudas sadias de variedades resistentes.

Uma das estratégias para solucionar este problema e outros, como o Mal-do-panamá (Fusarium oxysporum $\mathrm{f}$. sp. cubense), Sigatoka-amarela (Mycosphaerella musicola, Leach), Broca-do-rizoma [Cosmopolites sordidus (Germ.)] e nematóides, é a seleção de novos genótipos resistentes e/ou tolerantes e que apresentem boas características agronômicas, o que tem sido alcançado em Programas de Melhoramento da Bananeira (SILVA et al., 2000), possibilitando a obtenção de híbridos superiores, como Japira, FHIA 02, Preciosa, Pacovan Ken, Maravilha, Tropical e Thap Maeo, entre outros (BORGES; SOUZA, 2004).
Lima (2005) relata que antes de se recomendar a substituição de uma cultivar, faz-se necessário conhecer bem o novo genótipo, mediante estudos de caracterização e avaliação em diferentes ecossistemas, como altura das plantas, produtividade, resistência a doenças e precocidade, que influenciam diretamente no sistema de produção a ser escolhido.

Neste sentido, o objetivo foi avaliar o crescimento, desenvolvimento e produção de 23 genótipos de bananeiras no município de Goiânia-GO, visando selecionar os genótipos superiores para serem incorporados nos sistemas de produção comercial.

\section{MATERIAL E MÉTODOS}

O trabalho foi conduzido na Embrapa Transferência de Tecnologia - Escritório de Negócios de Goiânia-GO, com coordenadas de $16^{\circ} 38^{\prime} \mathrm{S}$ e $49^{\circ} 12^{\prime} \mathrm{W}$ e altitude de $736 \mathrm{~m}$, no período de janeiro de 2008 e novembro de 2009. O clima local, segundo a classificação de Köeppen, é do tipo Aw, quente e semi-úmido, com estação seca bem definida com chuvas predominantes na primavera e verão, de setembro a março. A temperatura máxima no período do trabalho alcançou $33,3{ }^{\circ} \mathrm{C}$ e a temperatura mínima a $11{ }^{\circ} \mathrm{C}$, com precipitação média anual de $1.514 \mathrm{~mm}$ e umidade relativa em torno de 70\% (UNIVERSIDADE FEDERAL DE GOIÁS, 2011).

As mudas micropropagadas, provenientes da Embrapa Mandioca e Fruticultura Tropical, foram transplantadas para o campo, em área previamente preparada, com o espaçamento de $2,5 \times 3,0 \mathrm{~m}$, totalizando $7,5 \mathrm{~m}^{2}$ planta $^{-1}$. No primeiro ciclo de produção, todos os genótipos receberam os seguintes tratos culturais: irrigação complementar sem uma lâmina definida, apenas para a fixação das mudas no campo, controle de plantas daninhas, retirada de folhas secas, eliminação do coração e corte do pseudocaule após a colheita. Foi realizada adubação orgânica com 10 litros de esterco bovino e adubação fosfatada (superfosfato simples) com $40 \mathrm{~g}$ de $\mathrm{P}_{2} \mathrm{O}_{5} \operatorname{cova}^{-1}$ no plantio. Trinta dias após o plantio foram realizadas adubação nitrogenada e potássica (20-00-20), com $45 \mathrm{~g}$ de N e $45 \mathrm{~g}$ de $\mathrm{K}_{2} \mathrm{O}$ planta $^{-1}$.

Os 23 genótipos de bananeira foram dispostos em blocos casualizados, com três repetições de seis plantas por parcela, sendo a área total da parcela de $45 \mathrm{~m}^{2}$. Os genótipos avaliados foram Caipira, Calipso, Bucaneiro, FHIA-02, FHIA-17, Thap Maeo, FHIA-01, FHIA-18, PA42-44, PA94-01, ST42-08 (Garantida), PV42-53, PV42-142, e PV79-34, PV94-01, PV42-81 (Vitória), YB42-21, YB42-03 e YB42-07, além das 
Tabela 1 - Características dos genótipos de bananeira avaliados em Goiânia-GO

\begin{tabular}{|c|c|c|}
\hline Genótipo & Grupo & Genealogia (Origem) \\
\hline Prata Anã & $\mathrm{AAB}$ & Cultivar (Santa Catarina) \\
\hline FHIA-01 (Maravilha) & AAAB & Híbrido de Prata Anã (FHIA) \\
\hline FHIA-18 & AAAB & Híbrido de Prata Anã (FHIA) \\
\hline PA42-44 & AAAB & Híbrido de Prata Anã (FHIA) \\
\hline PA94-01 & AAAB & Híbrido de Prata Anã - Embrapa \\
\hline Pacovan & $\mathrm{AAB}$ & Cultivar (Nordeste) \\
\hline Pacovan Ken (PV 42-43) & AAAB & Híbrido Pacovan (Embrapa) \\
\hline PV42-81 (Vitória) & AAAB & Híbrido de Pacovan (Embrapa) \\
\hline PV42-142 (Japira) & $\mathrm{AAAB}$ & Híbrido de Pacovan (Embrapa) \\
\hline PV79-34 & AAAB & Híbrido de Pacovan (Embrapa) \\
\hline PV94-01 & AAAB & Híbrido de Pacovan (Embrapa) \\
\hline Maçã & $\mathrm{AAB}$ & Cultivar (Brasil) \\
\hline YB42-21 & AAAB & Híbrido de Yangambi - tipo Maçã (Embrapa) \\
\hline YB42-03 & $\mathrm{AAAB}$ & Híbrido de Yangambi - tipo Maçã (Embrapa) \\
\hline YB42-07 & $\mathrm{AAAB}$ & Híbrido de Yangambi - tipo Maçã (Embrapa) \\
\hline Grande Naine & AAA & Cultivar Cavendish \\
\hline FHIA 02 & AAAA & Híbrido de Cavendish (FHIA) \\
\hline FHIA17 & AAAA & Híbrido tipo Cavendish \\
\hline Caipira & AAA & Cultivar (África Ocidental) \\
\hline Thap Maeo & $\mathrm{AAB}$ & Cultivar tipo Mysore (Tailândia) \\
\hline Calipso & AAAA & Híbrido High Gate (Jamaica) \\
\hline Bucanero & AAAA & Híbrido High Gate (Jamaica) \\
\hline ST42-08 (Garantida) & $\mathrm{AAAB}$ & Híbrido de Prata Comum (Embrapa) \\
\hline
\end{tabular}

cultivares tradicionais Pacovan, Prata Anã, Maçã e Grand Naine (Tabela 1).

As características avaliadas foram altura das plantas $(\mathrm{m})$, circunferência do pseudocaule $(\mathrm{cm})$, número de folhas vivas na floração e na colheita, número de dias para florescimento, número de dias de formação do cacho, número de pencas por cacho, massa do fruto $(\mathrm{g})$, massa de penca $(\mathrm{kg})$, número de frutos, comprimento dos frutos $(\mathrm{cm})$ e diâmetro dos frutos $(\mathrm{mm})$.

A altura da planta foi avaliada durante a emissão da inflorescência, com auxílio de uma trena semi-flexível posicionada desde o nível do solo até a roseta foliar (ponto de inserção do engaço no pseudocaule). Na determinação da circunferência do pseudocaule, foi utilizada uma fita métrica a $0,20 \mathrm{~m}$ acima do nível do solo.

As características número de dias do plantio à colheita, número de dias do plantio ao florescimento e número de dias do florescimento à colheita foram obtidas por meio do cálculo da diferença entre a data da colheita e a data do plantio, data da emissão da inflorescência e a data do plantio, e data da colheita à data da emissão da inflorescência, respectivamente.

$\mathrm{Na}$ contagem das folhas durante o florescimento e colheita, foram consideradas as totalmente abertas e que apresentaram área verde acima de $10 \%$.

As massas dos cachos foram obtidas através da pesagem dos cachos, desconsiderado o peso do engaço, sendo utilizada uma balança eletrônica digital. A massa das pencas foi obtida por meio da pesagem da segunda penca do cacho, na qual também foram realizadas as avaliações de frutos: comprimento e diâmetro, utilizando uma fita métrica e a massa média dos frutos.

Os dados foram submetidos à análise de variância (teste F) e as médias comparadas pelo teste de Scott-Knott a 5\%. As análises foram realizadas pelo programa ASSISTAT (SILVA; AZEVEDO, 2002). 


\section{RESULTADOS E DISCUSSÃO}

Os genótipos que apresentaram plantas com maiores alturas foram as cultivares Vitória, Japira e Tropical, com valores médios respectivamente de 3,15; 3,15 e 3,06 m, que não diferiram entre si a 5\% (Tabela 2). Santos et al. (2006) relataram que em uma cultivar comercial, é indesejável que a bananeira expresse valores muitos elevados em altura, pois pode dificultar a colheita e provocar tombamento da planta em decorrência de ventos fortes e ataques de nematóides e broca. Além disso, a altura da planta é um descritor importante, tanto do ponto de vista fitotécnico como genético, permitindo maior adensamento e, consequentemente, maiores produtividades. A maioria das plantas avaliadas foram classificadas como de porte médio, com a altura variando de 2,00 m a 3,50 m, o que segundo Santos et al. (2006) é a faixa de altura mais recomendada comercialmente, facilitando a colheita e os tratos culturais.

Tabela 2 - Valores médios de altura das plantas, circunferência do pseudocaule, número de folhas por planta no florescimento e na colheita de diferentes genótipos de bananeiras, em Goiânia-GO. (2008 /2009)

\begin{tabular}{|c|c|c|c|c|}
\hline Genótipos & Altura da planta (m) & $\begin{array}{l}\text { Circunferência do } \\
\text { pseudocaule }(\mathrm{cm})\end{array}$ & $\begin{array}{l}\mathrm{N}^{\mathrm{o}} \text { folhas no } \\
\text { florescimento }\end{array}$ & $\mathrm{N}^{\mathrm{o}}$ folhas na colheita \\
\hline Prata-anã $^{1}$ & $2,22 \mathrm{e}$ & $56,38 \mathrm{c}$ & $16,22 \mathrm{a}$ & $7,83 \mathrm{a}$ \\
\hline FHIA $01^{1}$ & $2,46 \mathrm{~d}$ & $61,25 \mathrm{~b}$ & $13,93 \mathrm{~b}$ & $3,80 \mathrm{c}$ \\
\hline FHIA $18^{1}$ & $2,00 \mathrm{f}$ & $52,66 \mathrm{c}$ & $12,72 \mathrm{c}$ & $7,05 \mathrm{a}$ \\
\hline PA $42-44^{1}$ & $2,27 \mathrm{e}$ & $56,26 \mathrm{c}$ & $15,65 \mathrm{a}$ & $7,18 \mathrm{a}$ \\
\hline PA $94-01^{1}$ & $2,51 \mathrm{~d}$ & $65,66 \mathrm{~b}$ & $12,05 \mathrm{c}$ & $4,42 \mathrm{c}$ \\
\hline Pacovan $^{2}$ & $2,92 \mathrm{~b}$ & $56,93 \mathrm{c}$ & $4,91 \mathrm{f}$ & $7,47 \mathrm{a}$ \\
\hline Pacovan Ken ${ }^{2}$ & $2,97 \mathrm{~b}$ & $55,98 \mathrm{c}$ & $11,8 \mathrm{c}$ & $4,00 \mathrm{c}$ \\
\hline Vitória $^{2}$ & $3,15 \mathrm{a}$ & $59,77 \mathrm{c}$ & $12,55 \mathrm{c}$ & $1,00 \mathrm{~d}$ \\
\hline Japira $^{2}$ & $3,15 \mathrm{a}$ & $58,22 \mathrm{c}$ & $10,92 \mathrm{~d}$ & $2,00 \mathrm{~d}$ \\
\hline PV $79-34^{2}$ & $2,75 \mathrm{c}$ & $63,72 \mathrm{~b}$ & $9,16 \mathrm{e}$ & $4,44 \mathrm{c}$ \\
\hline PV $94-01^{2}$ & $2,71 \mathrm{c}$ & $56,08 \mathrm{c}$ & $11,31 \mathrm{~d}$ & $6,66 \mathrm{a}$ \\
\hline Maça $\tilde{a}^{3}$ & $2,92 \mathrm{~b}$ & $65,33 \mathrm{~b}$ & $13,93 \mathrm{~b}$ & $4,75 \mathrm{c}$ \\
\hline Tropical $^{3}$ & $3,06 \mathrm{a}$ & $71,11 \mathrm{a}$ & $11,55 \mathrm{c}$ & $5,60 \mathrm{~b}$ \\
\hline YB $42-07^{3}$ & $2,81 \mathrm{c}$ & $62,08 \mathrm{~b}$ & $5,66 \mathrm{f}$ & $5,91 \mathrm{~b}$ \\
\hline YB $42-03^{3}$ & $2,72 \mathrm{c}$ & $58,83 \mathrm{c}$ & $11,16 \mathrm{~d}$ & $5,30 \mathrm{~b}$ \\
\hline Grand Naine ${ }^{4}$ & $2,13 \mathrm{f}$ & $60,41 \mathrm{c}$ & $13,73 \mathrm{~b}$ & $6,93 \mathrm{a}$ \\
\hline FHIA $02^{4}$ & $1,99 \mathrm{f}$ & $48,60 \mathrm{~d}$ & $11,83 \mathrm{c}$ & $6,27 \mathrm{~b}$ \\
\hline FHIA $17^{4}$ & $2,84 \mathrm{c}$ & $73,25 \mathrm{a}$ & $10,5 \mathrm{~d}$ & $6,38 \mathrm{~b}$ \\
\hline Caipira $^{5}$ & $1,96 \mathrm{f}$ & $44,66 \mathrm{~d}$ & $5,05 \mathrm{f}$ & $6,23 \mathrm{~b}$ \\
\hline Thap Maeo ${ }^{5}$ & $2,72 \mathrm{c}$ & $56,03 \mathrm{c}$ & $13,00 \mathrm{c}$ & $7,00 \mathrm{a}$ \\
\hline Garantida $^{5}$ & $2,98 \mathrm{~b}$ & $54,67 \mathrm{c}$ & $3,89 \mathrm{f}$ & $4,68 \mathrm{c}$ \\
\hline Calypso $^{5}$ & $2,27 \mathrm{e}$ & $56,66 \mathrm{c}$ & $10,00 \mathrm{e}$ & $4,00 \mathrm{c}$ \\
\hline Bucanero $^{5}$ & $2,49 \mathrm{~d}$ & $64,60 \mathrm{~b}$ & $12,64 \mathrm{c}$ & $5,44 \mathrm{~b}$ \\
\hline Média $^{1}$ & 2,29 & 58,44 & 14,11 & 6,06 \\
\hline Média ${ }^{2}$ & 2,94 & 58,45 & 10,11 & 4,26 \\
\hline Média $^{3}$ & 2,88 & 64,34 & 10,58 & 5,39 \\
\hline Média ${ }^{4}$ & 2,32 & 60,75 & 12,02 & 6,53 \\
\hline Média $^{5}$ & 2,48 & 55,32 & 8,92 & 5,47 \\
\hline $\mathrm{CV}(\%)$ & 3,76 & 5,14 & 8,13 & 16,96 \\
\hline
\end{tabular}

${ }^{1}$ Bananas do tipo Prata-Anã; ${ }^{2}$ Tipo Pacovan; ${ }^{3}$ Tipo Maçã; ${ }^{4}$ Cavendish; ${ }^{5}$ Outros tipos 
No grupo dos genótipos do tipo Pacovan, foram encontradas as maiores médias para os valores de altura, obtendo 2,94 m, no entanto, apenas os genótipos Vitória e Japira apresentaram valores dentro dos encontrados por Silva et al. (2003), que obtiveram plantas com altura superior a 3,00 m. Azevedo (2010) confirma o grande porte do grupo Pacovan, classificando-o como de porte alto, com variação de 3,52 a 4,21 m de altura.

Os genótipos Caipira, FHIA 02, FHIA 18 e Grand Naine, apresentaram plantas com porte menor, com altura média de 1,96; 1,99; 2,00 e 2,13 m, respectivamente (Tabela 2). Resultados superiores, nas mesmas condições de manejo, foram observados por Ramos et al. (2009), Silva et al. (2003) e Oliveira et al. (2007). Silva et al. (2003) avaliaram os mesmos genótipos e também não encontraram condições ambientais favoráveis para a expressão do porte.

Para circunferência do pseudocaule, verificou-se que o genótipo FHIA 17 (Grupo Cavendish) apresentou maior média em relação aos genótipos avaliados, com $73 \mathrm{~cm}$ de circunferência (Tabela 2). Silva e Alves (1999) relacionam a circunferência do pseudocaule com o vigor da planta, que se expressa também pela sua capacidade de sustentação do cacho. Por isso, genótipos que apresentam maior diâmetro são menos suscetíveis ao tombamento. $\mathrm{O}$ genótipo FHIA 17 apresentou alto índice de tombamento devido em parte por sua susceptibilidade ao ataque da broca-do-rizoma [Cosmopolites sordidus (Germ.)]. Assim, para garantir resistência ao tombamento, as plantas além de apresentarem maior circunferência do pseudocaule, também devem possuir maior tolerância a broca e a nematóides.

$\mathrm{Na}$ avaliação por grupo, as bananeiras do tipo Maçã apresentaram maior média de circunferência do pseudocaule $(64,34 \mathrm{~cm})$, destacando-se o genótipo Tropical, com 71,11 cm (Tabela 2). Resultados semelhantes foram verificados por Ramos et al. (2009), que observaram pseudocaules mais espessos em genótipos tetraplóides.

É importante ressaltar que o genótipo Tropical apresentou maior valor médio de altura e vigor, o que segundo Ramos et al. (2009), indica que a planta é capaz de sustentar o cacho apesar de apresentar porte elevado.

Em relação ao número de folhas vivas no florescimento, a cultivar Prata-anã e o genótipo PA 4244, ambas do grupo Prata-anã, apresentaram os maiores resultados, com valores médios de 16,22 e 15,65 folhas, respectivamente (Tabela 2). Segundo Alves (1997), a avaliação do caráter número de folhas no florescimento reflete o potencial produtivo da variedade, que depende da taxa fotossintética e da sua tolerância às doenças, como exemplo, a Sigatoka-amarela. O maior número de folhas no florescimento, considerado importante parâmetro na avaliação de genótipos (SILVA et al., 2000), sugere que o cacho poderá ter condições satisfatórias para o seu desenvolvimento.

A presença de mais de oito folhas na planta em fase de florescimento é um fator considerado como suficiente para o desenvolvimento normal do cacho (SOTO BALLESTERO, 1992), o que corrobora com os resultados encontrados neste trabalho, onde o genótipo Garantida e Caipira que apresentaram as menores médias de número de folhas no florescimento, 5,05 e 3,89, respectivamente, obtiveram as menores massas de cacho, $7,39 \mathrm{~kg}$ e $8,77 \mathrm{~kg}$, respectivamente.

O maior ou menor número de folhas vivas nas plantas na época da colheita pode indicar maior vida útil da folha ou resistência dos genótipos a doenças foliares, como as Sigatokas negra e amarela (OLIVEIRA et al., 2007). Neste trabalho, os genótipos Vitória e Japira (Tipo Pacovan) apresentaram perdas significativas no número de folhas, com perdas de 10,92 e 9,16 para 1,0 e 2,0 folhas, respectivamente (Tabela 2). Oliveira et al. (2008) verificaram que plantas que apresentavam baixo número de folhas na colheita do primeiro ciclo, nos demais ciclos apresentaram também as menores médias de folhas, o que pode ser um indicativo para a não recomendação da cultivar. Teixeira, Ruggieno e Natale (2001) concluíram que a senescência das folhas em bananeiras, entre a época da emissão da inflorescência e a colheita dos cachos, é acelerada em condições de desequilíbrio nutricional de $\mathrm{N}$ e K.

Em relação aos resultados referentes ao ciclo das plantas, o genótipo FHIA 17 foi o mais tardio dos avaliados, com a média de 571 dias do plantio até a colheita, no primeiro cacho. Já os genótipos PV 94-01 e Pacovan (Tipo Pacovan), FHIA 02 (Grupo Cavendish), FHIA 18 e PA 42-44 (Tipo Prata-anã), foram os mais precoces (Tabela 3). Observou-se que os valores encontrados neste trabalho diferem dos obtidos por outros autores, como Silva et al. (2003), em Cruz das Almas-BA, Ramos et al. (2009), em Botucatu-SP e Silva (2003), em Viçosa-MG, Lavras-MG, Guanambi-BA e Jaíba-MG, o que pode ser justificado pela influência direta das condições de ambiente, como, altitude, temperatura, umidade, entre outros, no ciclo da cultura (ALVES et al.,1995).

Segundo Silva et al. (2000), a precocidade é uma característica importante, especialmente, sob o ponto de vista econômico, pois resulta na obtenção de ciclos sucessivos de produção em menor espaço de tempo, aumentando a produção e a produtividade. É interessante para o produtor rural que a planta apresente precocidade, pois terá uma antecipação do seu investimento aplicado (RAMOS et al., 2009). 
Tabela 3 - Valores médios de número de dias do plantio ao florescimento, número de dias entre o florescimento e a colheita, e número de dias do plantio à colheita (ciclo vital) de genótipos de bananeiras. Goiânia-GO (2008/2009)

\begin{tabular}{|c|c|c|c|}
\hline Genótipos & $\begin{array}{l}\mathrm{N}^{\circ} \text { de dias do plantio ao } \\
\text { florescimento }\end{array}$ & $\begin{array}{l}\mathrm{N}^{\circ} \text { de dias do florescimento } \\
\text { à colheita }\end{array}$ & $\begin{array}{c}\mathrm{N}^{\circ} \text { de dias do plantio à } \\
\text { colheita }\end{array}$ \\
\hline Prata-anã $\tilde{a}^{1}$ & $349,33 \mathrm{c}$ & $139,08 \mathrm{~b}$ & $488,41 \mathrm{c}$ \\
\hline Fhia $01^{1}$ & $369,75 \mathrm{~b}$ & $122,58 \mathrm{c}$ & $492,33 \mathrm{c}$ \\
\hline FHIA $18^{1}$ & $300,03 \mathrm{e}$ & $135,48 \mathrm{~b}$ & $435,51 \mathrm{e}$ \\
\hline PA42-44 ${ }^{1}$ & $302,33 \mathrm{e}$ & $146,33 \mathrm{~b}$ & $448,66 \mathrm{~d}$ \\
\hline PA $94-01^{1}$ & $325,33 \mathrm{~d}$ & $131,45 \mathrm{c}$ & $456,78 \mathrm{~d}$ \\
\hline Pacovan $^{2}$ & $301,00 \mathrm{e}$ & $145,00 \mathrm{~b}$ & $446,00 \mathrm{~d}$ \\
\hline Pacovan Ken ${ }^{2}$ & $395,45 \mathrm{~b}$ & $150,66 \mathrm{~b}$ & 546,11 a \\
\hline Vitória² & $326,46 \mathrm{~d}$ & $164,07 \mathrm{a}$ & $490,50 \mathrm{c}$ \\
\hline Japira $^{2}$ & $328,08 \mathrm{~d}$ & $163,87 \mathrm{a}$ & $491,95 \mathrm{c}$ \\
\hline PV 79-34² & $316,33 \mathrm{~d}$ & $143,33 \mathrm{~b}$ & $459,66 \mathrm{~d}$ \\
\hline PV 94-01² & $276,67 \mathrm{e}$ & $147,10 \mathrm{~b}$ & $423,77 \mathrm{e}$ \\
\hline Maçã $\tilde{a}^{3}$ & $370,40 \mathrm{~b}$ & $103,70 \mathrm{~d}$ & $474,10 \mathrm{c}$ \\
\hline Tropical $^{3}$ & $375,39 \mathrm{~b}$ & $121,86 \mathrm{c}$ & $497,25 \mathrm{c}$ \\
\hline YB $42-07^{3}$ & 391,66 b & 165,34 a & $557,00 \mathrm{a}$ \\
\hline YB $42-03^{3}$ & $349,66 \mathrm{c}$ & $120,50 \mathrm{c}$ & $470,16 \mathrm{c}$ \\
\hline Grand Naine ${ }^{4}$ & $358,00 \mathrm{c}$ & $119,00 \mathrm{c}$ & $477,00 \mathrm{c}$ \\
\hline FHIA $02^{4}$ & $280,97 \mathrm{e}$ & $128,44 \mathrm{c}$ & $409,41 \mathrm{e}$ \\
\hline FHIA $17^{4}$ & 446,83 a & $124,72 \mathrm{c}$ & $571,55 \mathrm{a}$ \\
\hline Caipira $^{5}$ & $320,52 \mathrm{~d}$ & $107,03 \mathrm{~d}$ & $427,55 \mathrm{e}$ \\
\hline Thap $\mathrm{Maeo}^{5}$ & $347,30 \mathrm{c}$ & $111,53 \mathrm{~d}$ & $458,83 \mathrm{~d}$ \\
\hline Garantida $^{5}$ & $334,66 \mathrm{~d}$ & $124,67 \mathrm{c}$ & $459,33 \mathrm{~d}$ \\
\hline Calypso $^{5}$ & $336,33 \mathrm{~d}$ & $108,67 \mathrm{~d}$ & $445,00 \mathrm{~d}$ \\
\hline Bucanero $^{5}$ & $382,00 \mathrm{~b}$ & $131,66 \mathrm{c}$ & $513,66 \mathrm{~b}$ \\
\hline Média $^{1}$ & 329,35 & 134,98 & 464,34 \\
\hline Média $^{2}$ & 324,00 & 152,33 & 476,33 \\
\hline Média $^{3}$ & 371,78 & 127,85 & 499,63 \\
\hline Média $^{4}$ & 361,93 & 124,05 & 485,99 \\
\hline Média $^{5}$ & 344,16 & 116,71 & 460,87 \\
\hline $\mathrm{CV}(\%)$ & 4,76 & 8,36 & 3,86 \\
\hline
\end{tabular}

${ }^{1}$ Bananas do tipo Prata-Anã; ${ }^{2}$ Tipo Pacovan; ${ }^{3}$ Tipo Maçã; ${ }^{4}$ Cavendish; ${ }^{5}$ Outros tipos

Um caráter importante de se observar é o intervalo de tempo entre o florescimento e colheita do cacho, no qual se verificou que nem sempre as plantas mais precoces no florescimento são as mais precoces para a colheita. Segundo Lima et al. (2005), os genótipos que apresentaram os maiores ciclos do plantio à colheita, não foram os mesmos que demandaram maior tempo para florescer, mostrando com isto que, existem diferenças marcantes entre os genótipos em relação ao período que vai do florescimento à colheita. Essa observação é corroborada pelos resultados encontrados, onde o genótipo YB 42-07 apresentou uma variação de 165 dias do florescimento à colheita, valor bem superior à média encontrada no grupo maçã, que foi de 127 dias, apesar de não ter sido encontrada diferença significativa no número de dias para o florescimento (Tabela 3). Para a colheita, esse genótipo foi bem mais tardio do que os outros avaliados. 
Com relação às características referentes à produção, os genótipos FHIA 17, Grand Naine e Bucaneiro apresentaram maiores valores de massa do cacho 28,$55 ; 26,80$ e $25,30 \mathrm{~kg}$, respectivamente (Tabela 4). Apresentaram ainda maior quantidade e tamanho dos frutos, com exceção do genótipo Bucaneiro, que apresentou menor quantidade de frutos. Esses genótipos, mais produtivos, apresentaram ciclos maiores devido ao fato de possuírem cachos mais pesados, consequentemente, maior o tempo para formação e enchimento dos frutos.

Nos genótipos do tipo Pacovan, observouse cachos pouco produtivos, com $13 \mathrm{~kg}$ em média, e ciclo relativamente longo, este comportamento pode ser justificado pelo alto índice de quebramento do pseudocaule, fato este atribuído a excessiva altura e

Tabela 4 - Valores médios de massa de cacho (MC), massa de penca (MP), número de pencas (NP), número de frutos por cacho (NFC), massa média do fruto (MMF), comprimento (CF) e diâmetro de frutos (DF) em diferentes genótipos de bananeiras. Goiânia-GO (2008/2009)

\begin{tabular}{|c|c|c|c|c|c|c|c|}
\hline \multirow{2}{*}{ Genótipos } & \multirow{2}{*}{$\mathrm{MC}(\mathrm{kg})$} & \multirow{2}{*}{$\mathrm{MP}(\mathrm{kg})$} & \multirow{2}{*}{ NP } & \multicolumn{4}{|c|}{ Frutos } \\
\hline & & & & NFC & $\operatorname{MMF}(\mathrm{g})$ & $\mathrm{CF}(\mathrm{cm})$ & $\mathrm{DF}(\mathrm{mm})$ \\
\hline Prata-anã $^{1}$ & $18,88 \mathrm{c}$ & $17,65 \mathrm{c}$ & $6,83 \mathrm{e}$ & $87,16 \mathrm{~d}$ & $221,14 b$ & $22,54 \mathrm{a}$ & $35,46 \mathrm{c}$ \\
\hline Fhia $01^{1}$ & $22,15 \mathrm{~b}$ & $20,60 \mathrm{~b}$ & $6,93 \mathrm{e}$ & $96,66 \mathrm{c}$ & $252,03 \mathrm{a}$ & $23,24 \mathrm{a}$ & $41,53 \mathrm{a}$ \\
\hline FHIA $18^{1}$ & $13,94 \mathrm{e}$ & $12,87 \mathrm{e}$ & $8,27 \mathrm{c}$ & $101,97 \mathrm{c}$ & $144,58 \mathrm{~d}$ & $17,25 \mathrm{~b}$ & $35,60 \mathrm{c}$ \\
\hline PA42-44 ${ }^{1}$ & $13,11 \mathrm{f}$ & $11,53 \mathrm{f}$ & $6,63 \mathrm{e}$ & $76,33 \mathrm{e}$ & $158,30 \mathrm{~d}$ & $19,33 \mathrm{~b}$ & $36,66 \mathrm{c}$ \\
\hline PA $94-01^{1}$ & $18,69 \mathrm{c}$ & $16,85 \mathrm{c}$ & $8,66 \mathrm{c}$ & $124,58 \mathrm{~b}$ & $153,39 d$ & $18,93 \mathrm{~b}$ & $34,16 \mathrm{c}$ \\
\hline Pacovan $^{2}$ & $11,73 \mathrm{f}$ & $10,56 \mathrm{f}$ & $6,64 \mathrm{e}$ & $78,19 \mathrm{e}$ & $156,07 \mathrm{~d}$ & $19,21 \mathrm{~b}$ & $34,75 \mathrm{~d}$ \\
\hline Pacovan Ken ${ }^{2}$ & $12,35 \mathrm{f}$ & $11,40 \mathrm{f}$ & $5,45 \mathrm{f}$ & $63,86 \mathrm{f}$ & $190,85 \mathrm{c}$ & $19,58 \mathrm{~b}$ & $37,75 \mathrm{c}$ \\
\hline Vitória $^{2}$ & $12,43 \mathrm{f}$ & $11,43 \mathrm{f}$ & $5,88 \mathrm{f}$ & $73,71 \mathrm{e}$ & $207,40 \mathrm{~b}$ & $19,66 \mathrm{~b}$ & $36,66 \mathrm{c}$ \\
\hline Japira $^{2}$ & $12,01 \mathrm{f}$ & $10,92 \mathrm{f}$ & $5,96 \mathrm{f}$ & $68,78 \mathrm{f}$ & $150,54 \mathrm{~d}$ & $20,43 \mathrm{~b}$ & $33,23 \mathrm{c}$ \\
\hline PV 79-34² & $16,18 \mathrm{~d}$ & $14,70 \mathrm{~d}$ & $6,94 \mathrm{e}$ & $87,22 \mathrm{~d}$ & $166,33 \mathrm{~d}$ & $18,50 \mathrm{~b}$ & $36,86 \mathrm{c}$ \\
\hline PV 94-012 & $13,52 \mathrm{e}$ & $12,59 \mathrm{e}$ & $5,80 \mathrm{f}$ & $73,71 \mathrm{e}$ & $191,66 \mathrm{c}$ & $19,32 \mathrm{~b}$ & $38,55 \mathrm{~b}$ \\
\hline Maçã $\tilde{a}^{3}$ & $10,68 \mathrm{~g}$ & $9,36 \mathrm{~g}$ & $5,56 \mathrm{e}$ & $82,86 \mathrm{e}$ & $129,33 \mathrm{e}$ & $15,66 \mathrm{c}$ & $34,84 \mathrm{~d}$ \\
\hline Tropical $^{3}$ & $12,53 \mathrm{f}$ & $11,52 \mathrm{f}$ & $5,45 \mathrm{f}$ & $81,40 \mathrm{e}$ & $149,07 \mathrm{~d}$ & $18,01 \mathrm{~b}$ & $38,11 \mathrm{~b}$ \\
\hline YB $42-07^{3}$ & $11,41 \mathrm{f}$ & $10,44 \mathrm{f}$ & $6,83 \mathrm{e}$ & $87,25 \mathrm{~d}$ & $124,77 \mathrm{e}$ & $16,58 \mathrm{c}$ & $32,66 \mathrm{~d}$ \\
\hline YB $42-03^{3}$ & $10,45 \mathrm{~g}$ & $9,40 \mathrm{~g}$ & $5,80 \mathrm{f}$ & $84,80 \mathrm{~d}$ & $114,65 \mathrm{e}$ & $15,16 \mathrm{c}$ & $36,86 \mathrm{c}$ \\
\hline Grand Naine ${ }^{4}$ & $26,80 \mathrm{a}$ & $25,07 \mathrm{a}$ & $7,76 \mathrm{~d}$ & $124,43 \mathrm{~b}$ & $235,84 \mathrm{a}$ & $25,00 \mathrm{a}$ & $38,61 \mathrm{~b}$ \\
\hline FHIA $02^{4}$ & $12,48 \mathrm{f}$ & $11,46 \mathrm{f}$ & $7,97 \mathrm{~d}$ & $91,62 \mathrm{~d}$ & $144,20 \mathrm{~d}$ & $17,85 \mathrm{~b}$ & $35,40 \mathrm{c}$ \\
\hline FHIA $17^{4}$ & $28,55 \mathrm{a}$ & $26,16 \mathrm{a}$ & $9,60 \mathrm{~b}$ & $146,10 \mathrm{a}$ & $214,00 \mathrm{~b}$ & $23,16 \mathrm{a}$ & $36,90 \mathrm{c}$ \\
\hline Caipira $^{5}$ & $7,85 \mathrm{~g}$ & $7,39 \mathrm{~g}$ & $6,06 \mathrm{f}$ & $100,45 \mathrm{c}$ & $78,01 \mathrm{f}$ & $13,10 \mathrm{c}$ & $31,11 \mathrm{e}$ \\
\hline Thap $\mathrm{Maeo}^{5}$ & $16,80 \mathrm{~d}$ & $15,72 \mathrm{~d}$ & $10,37 \mathrm{a}$ & $142,91 \mathrm{a}$ & $113,47 \mathrm{e}$ & $14,66 \mathrm{c}$ & $36,21 \mathrm{c}$ \\
\hline Garantida $^{5}$ & $9,75 \mathrm{~g}$ & $8,77 \mathrm{~g}$ & $5,8 \mathrm{f}$ & $69,41 \mathrm{f}$ & $131,38 \mathrm{e}$ & $17,83 \mathrm{~b}$ & $33,60 \mathrm{~d}$ \\
\hline Calypso $^{5}$ & $12,35 \mathrm{f}$ & $11,38 \mathrm{f}$ & $6,50 \mathrm{e}$ & $98,50 \mathrm{c}$ & $120,55 \mathrm{e}$ & $24,25 \mathrm{a}$ & $28,50 \mathrm{f}$ \\
\hline Bucanero $^{5}$ & $25,30 \mathrm{a}$ & $23,81 \mathrm{a}$ & $6,86 \mathrm{e}$ & $76,33 \mathrm{c}$ & $252,06 \mathrm{a}$ & $25,62 \mathrm{a}$ & $40,17 \mathrm{a}$ \\
\hline Média $^{1}$ & 17,35 & 15,90 & 7,46 & 102,47 & 185,89 & 20,26 & 36,68 \\
\hline Média $^{2}$ & 13,04 & 11,93 & 6,11 & 72,01 & 177,14 & 19,45 & 36,30 \\
\hline Média $^{3}$ & 11,27 & 10,18 & 5,91 & 84,07 & 129,46 & 16,35 & 35,62 \\
\hline Média ${ }^{4}$ & 22,61 & 20,90 & 8,44 & 112,72 & 198,01 & 22,00 & 36,97 \\
\hline Média $^{5}$ & 14,41 & 13,41 & 7,12 & 97,52 & 139,09 & 19,09 & 33,92 \\
\hline $\mathrm{CV}(\%)$ & 10,53 & 11,00 & 5,15 & 5,15 & 7,74 & 9,15 & 3,96 \\
\hline
\end{tabular}

${ }^{1}$ Bananas do tipo Prata-Anã; ${ }^{2}$ Tipo Pacovan; ${ }^{3}$ Tipo Maçã; ${ }^{4}$ Cavendish; ${ }^{5}$ Outros tipos 
baixa espessura do pseudocaule do grupo. O genótipo FHIA 17 foi o mais produtivo dentre os demais avaliados, destacando-se na maioria dos caracteres produtivos, sendo inferior aos outros genótipos apenas nos caracteres massa média e diâmetro do fruto, o que pode ser atribuído ao elevado índice de tombamento provocado pela alta susceptibilidade à broca-do-rizoma, no qual as plantas tombavam antes do ponto de colheita, com os frutos ainda magros.

Em relação ao número de pencas, Flores (2000) salienta que esse caráter é de grande interesse para o produtor e fundamental para o melhoramento genético da bananeira, uma vez que a penca constitui-se na unidade comercial. $\mathrm{O}$ genótipo Thap Maeo foi destaque com a média de 10,37 pencas, seguida da FHIA 17, com 9,60 pencas (Tabela 4). Resultados semelhantes obtidos com a cultivar Tap Maeo foram obtidos por Braga Filho et al. (2011). Os genótipos do tipo Maçã e Pacovan foram os que apresentaram menores médias, 5,91 e 6,11 , respectivamente, sendo esses os genótipos menos produtivos, o que demonstra uma possível relação positiva entre o número de frutos e massa dos cachos, semelhantes aos resultados obtidos por Lima Neto et al. (2003), confirmando que o número de frutos é importante para o tamanho e massa do cacho (SILVA; ALVES, 1999).

Em relação ao comprimento dos frutos, a Grand Naine apresentou frutos mais compridos que as demais variedades $(25 \mathrm{~cm})$, resultados semelhantes foram encontrados por Ramos et al. (2009) e Donato et al. (2006). Outros genótipos que também se destacaram em relação ao comprimento dos frutos, foram FHIA 17, FHIA 01 e a cultivar Prata-anã.

Os genótipos FHIA 01 e Bucaneiro apresentaram os maiores valores referentes ao diâmetro dos frutos, 41,53 e 40,17 mm, respectivamente (Tabela 4), o que influencia diretamente na massa média dos frutos, e consequentemente na massa do cacho, na qual foi observado nesses genótipos uma das maiores médias.

Dentre os genótipos do grupo Prata-anã, verificouse que todos apresentaram características adequadas de crescimento, porém, no que diz respeito à produção, os genótipos FHIA 01, Prata-anã e PA 94-01, se destacaram apresentando cachos mais pesados.

Entre os genótipos do tipo Pacovan, observouse que os genótipos mais promissores foram PV 79-34 e PV 94-01, com massa de cacho de 16,18 e 13,52 kg, respectivamente, superiores aos demais genótipos e até mesmo superior à variedade Pacovan (Tabela 4). O genótipo PV 79-34 apresentou altura e circunferência do caule adequado para a sustentação do cacho. $\mathrm{O}$ genótipo Pacovan Ken por apresentar cachos pequenos e ciclo tardio não foi considerado adequado para o cultivo em Goiânia.

As bananeiras do Tipo Maçã, o genótipo Tropical apresentou crescimento adequado, isto é, boa relação entre altura $(3,06 \mathrm{~m})$ e circunferência do pseudocaule $(71,11 \mathrm{~m})$, além de bons aspectos produtivos de massa de cacho, massa de fruto e comprimento e diâmetro dos frutos, $12,53 \mathrm{~kg}, 149,07 \mathrm{~g}, 18,01 \mathrm{~cm}$ e $38,11 \mathrm{~mm}$, respectivamente.

No grupo das bananas Cavendish, observou-se que o genótipo FHIA 17 apresentou bons parâmetros de crescimento e produção, altura $(2,84 \mathrm{~m})$, circunferência do pseudocaule $(73,25 \mathrm{~cm})$, massa de cacho $(28,55 \mathrm{~kg})$, números de frutos (146) e massa média de frutos (214 g), sendo prejudicada apenas pela alta susceptibilidade à Broca-do-rizoma e pelo ciclo tardio. O genótipo FHIA 02 não apresentou boas características de crescimento nem de produção.

Entre os demais grupos de genótipos avaliados, a Bucaneiro apresentou as melhores características produtivas, produzindo boa massa de cacho $(25,30 \mathrm{~kg})$ e bons parâmetros de crescimento, $2,49 \mathrm{~m}$ de altura e $64,60 \mathrm{~cm}$ de circunferência do pseudocaule. A Caipira foi o genótipo que apresentou o menor porte, com 1,96 m. A cultivar Prata-anã e o genótipo PA 42-44 apresentaram plantas com maior número de folhas vivas no florescimento e colheita.

O genótipo FHIA 02 foi o mais precoce dos avaliados, porém, apresentou baixa massa de cacho $12,48 \mathrm{~kg}$. Já o FHIA 17 foi o mais tardio (571 dias do plantio à colheita), no entanto, apresentou um bom número de pencas $(9,6)$, o maior número de frutos por cacho e a maior massa de cacho, sendo o destaque em produtividade, seguido pelas cultivares Grand Naine e Bucaneiro e o genótipo FHIA 01.

\section{CONCLUSÃo}

As cultivares FHIA 17, Tropical, FHIA 01, Grand Naine e Bucaneiro pelos melhores desempenhos fenológicos e produtivos foram qualificadas como promissoras para serem incorporadas ao sistema de produção do bananicultor em Goiás.

\section{REFERÊNCIAS}

ALVES, E. J.; OLIVEIRA, M. A. Práticas culturais. In: ALVES, E. J. (Org.). A cultura da banana: aspectos técnicos, socioeconômicos e agroindustriais. 2. ed. rev. Brasília: Embrapa-SPI; Cruz das Almas: Embrapa-CNPMF, 1997. p. 335-352. 
ALVES, E. J. et al. Caracterização e avaliação de germoplasma de banana (Musa spp.). In: Congresso Brasileiro de Fruticultura, 1984, Florianópolis: SBF/ Empasc, 1984. p. 202-212.

AZEVEDO, V. F. de. Avaliação de bananeiras do tipo prata, de porte alto, no semiárido. 2010. 79 f. Dissertação (Mestrado em Produção Vegetal no Semiárido) - Universidade Estadual de Montes Claros, Janaúba, 2010.

BRAGA FILHO, J. R. et al. Lâminas de irrigação e genótipos na produção e qualidade de frutos de bananeira. Pesquisa Agropecuária Tropical, v. 41, n. 2, p. 155-162, 2011.

BORGES, A. L.; SOUZA, L. S. O cultivo da bananeira. Cruz das Almas: Embrapa Mandioca e Fruticultura, 2004. 279 p.

CORDEIRO, Z. J. M. et al. 'Preciosa': variedade de banana resistente à sigatoka-negra, sigatoka-amarela e mal-do-panamá. Fitopatologia Brasileira, v. 30, n. 3, p. 316, 2005.

DONATO, S. L. R. et al. Comportamento de variedades e híbridos de bananeira (Musa spp.), em dois ciclos de produção no sudoeste da Bahia. Revista Brasileira de Fruticultura, v. 28, n. 1, p.139-144, 2006.

FLORES, J. C. de O. Avaliação de cultivares e híbridos de bananeira (Musa spp.) em quatro ciclos de produção em Cruz das Almas-BA. 2000. 109 f. Dissertação (Mestrado em Ciências Agrárias) - Universidade Federal da Bahia, Cruz das Almas, 2000.

GASPAROTTO, L.; PEREIRA, J.C.R. (Ed.). A Cultura da bananeira na região Norte do Brasil. Brasília: Embrapa Informação Tecnológica, 2010. 310 p.

INSTITUTO BRASILEIRO DE GEOGRAFIA E ESTATÍSTICA. 2009. Censo Agropecuario 2011. Disponível em: <http: //www.ibge.gov.br>. Acesso em: 18 maio 2011.

LIMA, M. B. et al. Avaliação de cultivares e híbridos de bananeira no recôncavo baiano. Ciência e Agrotecnologia, v. 29, n. 3 , p. $515-520,2005$.

LIMA NETO, F. P. et al. Relação entre caracteres de rendimento e desenvolvimento em genótipos de bananeira. Magistra, v. 15, n. 2, p. 275-281, 2003.

MOREIRA, R. S.; SAES, L. A. Considerações sobre o banco de germoplasma do IAC. In: CONGRESSO BRASILEIRO DE FRUTICULTURA, 7., 1984. Florianópolis: SBF/Empasc, 1984. p. $220-236$. v. 1 .

OLIVEIRA, C. A. O. et al. Genótipos de bananeiras em três ciclos na Zona da Mata Mineira. Revista Pesquisa Agropecuária Brasileira, v. 42, n. 2, p. 173-181, 2007.
OLIVEIRA, T. K. et al. Características agronômicas de genótipos de bananeiras em três ciclos de produção em Rio Branco, AC. Revista Pesquisa Agropecuária Brasileira, v. 43, n. 8, p. 1003-1010, 2008.

RAMOS, D.P. et al. Avaliação de genótipos de bananeira em Botucatu-SP. Revista Brasileira de Fruticultura, v.31, n.4, p.1092-1101, 2009.

SANTOS, S. C. et al. Caracterização morfológica e avaliação de cultivares de bananeira resistentes a sigatoka-negra (Mycosphaerella fijiensis Morelet) no sudoeste goiano. Revista Brasileira de Fruticultura, v. 28, n. 3, p. 449-453, 2006.

SILVA, F. A. S. E.; AZEVEDO, C. A. V. Versão do programa computacional Assistat para o sistema operacional Windows. Revista Brasileira de Produtos Agroindustriais, v. 4, n. 1, p. 71-78, 2002.

SILVA, S. de O.; BOLIANI, A. C.; CORRÊA, L. S. Avaliação de cultivares de bananeira (Musa spp.) na Região de Selvíria-MS. Revista Brasileira de Fruticultura, v. 28, n. 1, p. 101-103, 2006.

SILVA, S. de O. Programa de melhoramento de bananeira no Brasil - resultados recentes. Cruz das Almas: Embrapa Mandioca e Fruticultura, 2003. 36 p. (Documentos, 123).

SILVA, S. de O. et al. Avaliação de genótipos de bananeiras em diferentes ambientes. Revista Ciência e Agrotecnologia, v. 27, n. 4, p. 737-748, 2003.

SILVA, S. de O. et al. Caracterização morfológica e avaliação de cultivares e híbridos de bananeira. Revista Brasileira de Fruticultura, v. 22, p. 161-169, 2000.

SILVA, S. O.; ALVES, E. J. Melhoramento genético e novas cultivares de banana. Informe Agropecuário, v. 20, n. 196, p. 91-96, 1999.

SOTO BALlESTERO, M. Bananos: cultivo y comercialización. 2. ed. San José: Litografía e Imprenta Lil, 1992. $674 \mathrm{p}$.

TEIXEIRA, L. A. J.; RUGGIERO, C.; NATALE, W. Manutenção de folhas ativas em bananeiras - 'Nanição' por meio do manejo das adubações nitrogenada e potássica e da irrigação. Revista Brasileira de Fruticultura, v. 23, n. 3, 2001.

UNIVERSIDADE FEDERAL DE GOIÁS/Escola de Agronomia. Disponível em: <http://www.agro.ufg.br>. Acesso em: 18 maio 2011. 